\title{
Tocilizumab Association with Emerging Multi-drug Resistance Organisms and Mortality in critically ill patients with Coronavirus disease 2019 (COVID-19): A Multicenter, Retrospective Cohort Study
}

Ohoud Aljuhani ( $\sim$ Oaljuhani@kau.edu.sa )

King Abdulaziz University

Khalid Al Sulaiman

King Abdulaziz Medical City

Adel Alshabasy

King Abdulaziz University

Khalid Eljaaly

King Abdulaziz University

Abdulrahman I. Al Shaya

King Abdulaziz Medical City

Hyatham Albossiry

King Abdulaziz University

Mohammed Abodeif

King Abdulaziz University

\section{Bodoor Al Dosari}

King Abdulaziz University Hospital

\section{Amina Alkhalaf}

King Abdulaziz University

\section{Ghazwa Korayem}

Princess Nourah Bint Abdulrahman University

Muneera M. Aleissa

Princess Nourah Bint Abdulrahman University

Hisham A. Badreldin

King Abdulaziz Medical City

Shmeylan Al Harbi

King Abdulaziz Medical City

Abdullah Alhammad

King Saud University

\section{Ramesh Vishwakarma}

King Abdullah International Medical Research Center 


\section{Research Article}

Keywords: COVID-19, SARS-Cov-2, Tocilizumab, Secondary infection, CRE, Critically ill, Intensive Care Units (ICUs), Immunomodulatory drugs

Posted Date: May 10th, 2021

DOI: https://doi.org/10.21203/rs.3.rs-469073/v1

License: (c) (1) This work is licensed under a Creative Commons Attribution 4.0 International License. Read Full License 


\section{Abstract}

\section{Background:}

Tocilizumab is an IgG1 class recombinant humanized monoclonal antibody that directly inhibits IL-6 receptor. Several randomized clinical trials (RCTs) have evaluated tocilizumab's safety and efficacy in patients with COVID-19. These studies demonstrated conflicting results regarding tocilizumab's efficacy and safety. Our study aim is to determine the association between treatment with tocilizumab and emergence of multidrug-resistant bacteria and its effect on mortality in critically ill patients with Coronavirus disease 2019 (COVID-19).

\section{Methods:}

A multicenter, retrospective, cohort study conducted at two governmental tertiary hospitals in Saudi Arabia. All critically ill patients who were admitted to intensive care units (ICUs) with a positive COVID-19 PCR test between March $1^{\text {st, }} 2020$ and January 31 ${ }^{\text {st }}, 2021$ were included. Patients who received tocilizumab were compared to patients who did not receive it.

\section{Results:}

A total of 738 patients met our inclusion criteria and were included in the analysis. Of these 262 (35.5\%) received tocilizumab and 476 (64.5\%) were included in the control group. Patients who received tocilizumab did not have higher odds for the microbial isolation (OR 1.34; 95\% Cl, 0.91-1.94 p = 0.13), development of resistant organisms (OR 1.00; $95 \% \mathrm{Cl}, 0.51-1.98 \mathrm{p}=0.99)$, or detection of CarbapenemResistant Enterobacteria (CRE) (OR 0.67; 95\% Cl, 0.29-1.54 p = 0.34). In a multivariable logistic regression adjusting for possible cofounders, there was no difference in 30-day ICU mortality (OR $0.96 ; 95 \% \mathrm{Cl}, 0.65$ $1.43 \mathrm{p}=0.85$ ) or in-hospital mortality (OR 1.18; $95 \% \mathrm{Cl}, 0.79-1.76 \mathrm{p}=0.42)$. However, there was a significant difference in the incidence of respiratory failure requiring $\mathrm{MV}$ between the two groups (OR $2.27 ; 95 \% \mathrm{Cl}, 1.05-4.89 \mathrm{p}=0.03)$.

\section{Conclusions:}

Tocilizumab use in critically ill COVID-19 patients was not associated with microbial isolation, emergence of resistant organisms, detection of CRE organisms, or mortality benefits. However, tocilizumab use was associated with an increased risk of respiratory failure requiring mechanical ventilation.

\section{Background}

With the emergence of the novel coronavirus (SARS-CoV-2) in December 2019 in Wuhan, China (1), the world has been dealing with a new highly contagious disease. As of this 04/27/2021, the novel coronavirus disease 2019 (COVID-19) has infected over 148 million people worldwide, with mortality exceeding 3.1 million people (1). Often times, patients hospitalized with COVID-19 present with pneumonia due to excessive host immune response causing an acute respiratory distress syndrome (2). 
This respiratory distress has been associated with increased intensive care unit (ICU) admission and mortality (3).

The occurrence of severe respiratory distress in critically ill patients is attributed to a state known as the cytokine release syndrome (CRS), where the body produces pro-inflammatory cytokines (TNF-a, IL-1 $1 \beta$, IL-2, and IL-6) and chemokines (IL-8) (4). Recent studies have shown that there are increased levels of cytokines, specifically interleukin 6 (IL-6) in critically ill patients with COVID-19 (3). This suggests that elevated IL-6 may be a marker for poor prognosis in patients with COVID-19 (4). Thus, the use of therapeutic agents targeting IL-6, such as tocilizumab, in critically ill patients has been investigated $(5,6,7,12)$

Tocilizumab is an IgG1 class recombinant humanized monoclonal antibody that directly inhibits IL-6 receptor (9). Several randomized clinical trials (RCTs) have evaluated tocilizumab's safety and efficacy in patients with COVID-19 $(5,6,7,10,11)$. These studies demonstrated conflicting results regarding tocilizumab's efficacy. A randomized controlled trial that included 389 COVID-19 pneumonia patients who did not receive mechanical ventilation (MV) reported a decrease in the likelihood of the progression to MV or death when compared to standard therapy by day-28(6). The benefit of tocilizumab was not demonstrated in randomized controlled trials that included mild to moderate patients with COVID-19 pneumonia. Additionally, tocilizumab was well tolerated compared to standard therapy in most studies $(6,7,10)$.

Although tocilizumab was associated with a lower incidence of serious infections (11), new evidence emerged portraying an increased risk of new Delhi Metallo-Beta-Lactamase-Producing CarbapenemResistant Enterobacteria (NDM-CRE) acquisition in patients with COVID-19 (13). The incidence and risk factors for the development of resistant co-infections among patients who receive tocilizumab for COVID19 was not adequately assessed in previous studies. Thus, we sought to evaluate the incidence of multidrug-resistant bacteria, and its effect on mortality among critically ill patients with COVID-19 who received tocilizumab.

\section{Methods}

\section{Study design}

This was a multicenter retrospective, cohort study evaluating the risk of developing multi-drug resistance organisms (MDRO) in adult COVID19 critically ill patients (aged $\geq 18$ years) who received tocilizumab between March 01, 2020 and January 31, 2021. COVID-19 was diagnosed using reverse transcriptasepolymerase chain reaction (RT-PCR) obtained from nasopharyngeal or throat swabs. Patients were excluded if the ICU length of stay (LOS) was less than a day or if they had a "Do-Not-Resuscitate" code status within 24 hours of ICU admission.

Eligible patients were classified into two groups based on tocilizumab use during ICU stay. Bacteria and fungus were identified in the blood, urine, wound, drainage, cerebrospinal fluid (CSF), and respiratory 
specimens. Microbial isolates defined as sputum or endotracheal aspiration shows a growth of $\geq$ $100,000 \mathrm{CFU} / \mathrm{mL}$; bronchoalveolar lavage (BAL) shows a growth of $\geq 10,000 \mathrm{CFU}$ of single organism $/ \mathrm{mL}$ for protected specimen brushes (PSBs), and $\geq 100,000 \mathrm{CFU}$ of single organism/mL for BAL fluid. Additionally, urine cultures were considered significant if showing a growth of $\geq 100,000 \mathrm{CFU} / \mathrm{mL}$ of no more than two species of microorganisms ${ }^{13}$. Cultures were excluded if the laboratory reported them as a contaminant sample.

\section{Setting}

This study was conducted in two tertiary governmental hospitals; King Abdulaziz University Hospital, Jeddah (primary site) and was approved by Unit of Biomedical Ethics Research Committee at faculty of medicine in King Abdulaziz University with Reference No 15-21. The second site was King Abdulaziz Medical City, Riyadh and was approved by King Abdullah International Medical Research Center in February 2021 (Ref.\#NRC21/025/01).

\section{Data collection}

Demographic data, comorbidities, vital signs and laboratory tests, severity scores (i.e., Acute Physiology and Chronic Health Evaluation II (APACHE II), Sequential Organ Failure Assessment (SOFA) scores), Glasgow Coma Score (GCS), acute kidney injury (AKI), the needs for MV and MV parameters (e.g., $\mathrm{PaO}_{2} / \mathrm{FiO}_{2}$ ratio, $\mathrm{FiO}_{2}$ requirement) within 24 hours of $\mathrm{ICU}$ admission were collected from the electronic health record (See additional file 1). Additionally, renal profile, liver function tests (LFTs), coagulation profile (i.e., INR, aPTT, fibrinogen), and inflammatory markers (CRP, procalcitonin) within 24 hours of ICU admission were collected. Moreover culture information, including the presence of resistant organisms were collected during the ICU stay. Tocilizumab and systemic corticosteroids use were recorded for the eligible patients. All patients were followed until they were discharged from the hospital or died during the in-hospital stay, whichever occurred first.

\section{Outcomes}

The primary outcomes were to evaluate the incidence of microbial isolation, resistant organisms, and carbapenem-resistant Enterobacteriaceae (CRE) in COVID-19 critically ill patients who received tocilizumab. Secondary outcomes included 30-day ICU mortality, in-hospital mortality, hospital LOS, ICU LOS, MV duration, and ICU-related complications during ICU stay (i.e. acute kidney injury, acute liver injury, respiratory failure requires $\mathrm{MV}$, thrombosis/infraction).

\section{Definition (s)}

- Multidrug-resistant (MDR) is non-susceptibility to at least three or more agents, extensively drugresistant (XDR) is non-susceptibility to at least one agent in all but two or fewer antimicrobial categories remain susceptible, pandrug-resistant (PDR) is non-susceptibility to all agents in all antimicrobial categories. Susceptibility of gram-positive/negative bacteria created using documents and breakpoints based on Clinical Laboratory Standards Institute (CLSI) $(15,18)$. 
- Carbapenem-resistant Enterobacteriaceae (CRE) has been defined as carbapenem-nonsusceptible and extended-spectrum cephalosporin-resistant Escherichia coli, Enterobacter aerogenes, Enterobacter cloacaecomplex, Klebsiella pneumoniae, or Klebsiella oxytoca that may be secondary to metallo-betalactamases,zinc metallo-enzymes (e.g. New Dehli Metallo (NDM),VIM-1, IMP-1), AmpC beta-lactamase, as well as oxacillinases (e.g.OXA-23-like, OXA-48, OXA-58-like OXA-48) (14).

- Acute kidney injury (AKI) was defined using Acute Kidney Injury Network (AKIN) definition (16).

- Thrombosis/infraction was defined using the International Classification of Diseases, Tenth Revision, Clinical Modification (ICD10-CM) code (17).

- Respiratory failure was defined as one of following types; hypoxemic respiratory failure with $\mathrm{PaO} 2<$ $60 \mathrm{~mm} \mathrm{Hg}$ and normal or low arterial carbon dioxide tension or hypercapnic respiratory failure ( $\mathrm{PaCO} 2>50 \mathrm{~mm} \mathrm{Hg}$ ) that requires mechanical ventilation (18).

- Acute liver injury was defined as alanine aminotransferase (ALT) exceeding three times the upper limit of normal or double in patients with elevated baseline ALT(18).

\section{Data management and Statistical analysis}

Categorical data were expressed as number and percentage, continuous variables were expressed as mean and standard deviation (SD), or median and lower quartile (Q1) and upper quartile (Q3), as appropriate.

Categorical variables were analyzed using Chi-square or Fisher's exact test, and continuous variables were analyzed using student t-test or Mann-Whitney $U$ test, as appropriate. A multivariable logistic regression was used to evaluate the primary outcomes after adjusting for possible co-founders including patient's comorbidities (i.e. diabetes mellitus (DM), chronic kidney disease (CKD) on dialysis), history of hospitalization or invasive procedure (surgery) within 1 year, history of antibiotics exposure in the last 3 months, systemic corticosteroids use during ICU and ICU LOS. For the secondary outcomes, a multivariable logistic and generalized linear regression were used to assess the relationship between tocilizumab use and the different outcomes considered in this study after adjusting for the patients' age, SOFA score, $\mathrm{PaO} 2 / \mathrm{FiO} 2$ ratio baseline and systemic corticosteroids during ICU. The odds ratios (OR) and estimates with the $95 \%$ confidence intervals $(\mathrm{Cl})$ were reported for the associations.

We assessed model fit using the Hosmer-Lemeshow goodness-of-fit test. No imputation was made for missing data as the cohort of patients in our study was not derived from random selection. We considered a $p$-value of $<0.05$ to be statistically significant. All statistical analyses were carried using SAS version 9.4 for all statistical analyses.

\section{Results}

A total of 738 patients met our inclusion criteria and were included in the analysis. Of these 262 (35.5\%) received tocilizumab and $476(64.5 \%)$ did not receive tocilizumab and were included in the control group. 
The median (Q1, Q3) dose of tocilizumab given per day was $400 \mathrm{mg}(400,600), 39.1 \%$ of the patients received tocilizumab early within 24 hours of ICU admission.

\section{Demographic and Clinical Characteristics}

For all patients, the mean (SD) age was 61 years (14.7), with the majority of patients being male (72.1\%). There were some notable differences between the two groups. Patients who received tocilizumab were younger in age (59.2 vs. 61.9 years, $p=0.014)$, had higher C-reactive protein (CRP) (167 vs. $143, p<$ $0.001)$, had lower median $\mathrm{PaO}_{2} / \mathrm{FiO}_{2}$ ratio within 24 hours of admission $(75.5$ vs. $87.8, \mathrm{p}<0.001)$ and were more likely to have received systemic corticosteroid use in the ICU $(93.4 \%$ vs. $84.9 \%, p<0.001)$. Patients who received tocilizumab were less likely to have a history of hospitalization or surgery within a year $(7.4 \%$ vs. $20.5 \%, p<0.001)$ compared with the control group, respectively (Additional file 1 - Table 1 ). Additionally, prevalence of diabetes, chronic kidney disease, and ischemic heart disease were significantly lower in the tocilizumab group compared with the control group (Additional file 2 - Table 2).

Table 1

Regression analysis for the primary outcomes

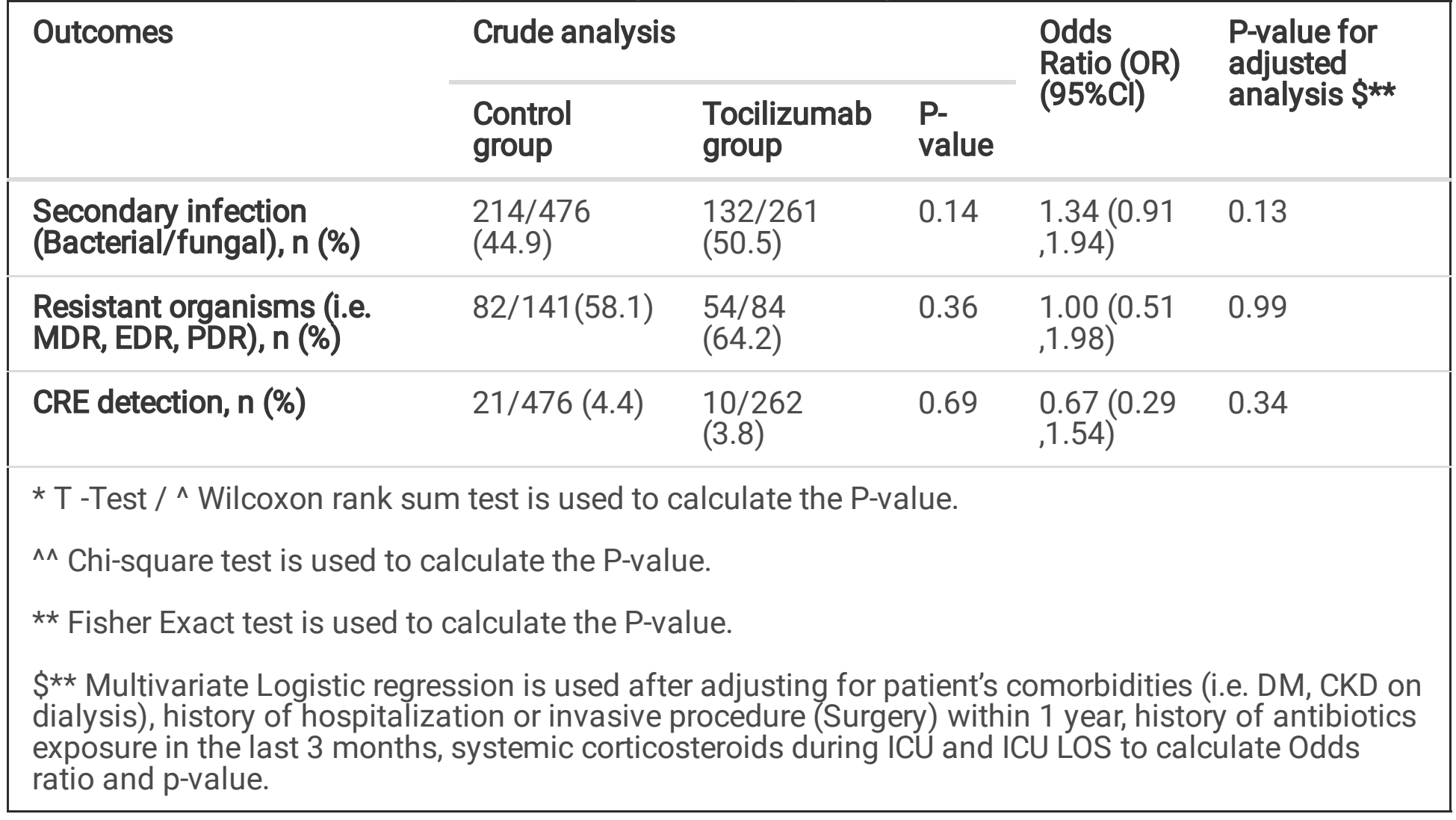


Table 2

Regression analysis for secondary outcomes

\begin{tabular}{|c|c|c|c|c|c|}
\hline \multirow[t]{2}{*}{ Outcomes } & \multicolumn{3}{|c|}{ Crude analysis } & \multirow{2}{*}{$\begin{array}{l}\text { Odds Ratio } \\
\text { (OR) (95\%Cl) }\end{array}$} & \multirow{2}{*}{$\begin{array}{l}\text { P-value for } \\
\text { adjusted } \\
\text { analysis } \$ \star \star\end{array}$} \\
\hline & $\begin{array}{l}\text { Control } \\
\text { group }\end{array}$ & $\begin{array}{l}\text { Tocilizumab } \\
\text { group }\end{array}$ & $\begin{array}{l}\mathrm{P}- \\
\text { value }\end{array}$ & & \\
\hline In-hospital mortality, n (\%) ${ }^{\Delta}$ & $\begin{array}{l}210 / 447 \\
(46.9)\end{array}$ & $\begin{array}{l}116 / 259 \\
(44.7)\end{array}$ & 0.57 & $\begin{array}{l}1.18(0.791 \\
, 1.761)\end{array}$ & 0.42 \\
\hline 30 day- ICU mortality, $\mathrm{n}(\%){ }^{\Delta}$ & $\begin{array}{l}187 / 449 \\
(41.6)\end{array}$ & $\begin{array}{l}93 / 260 \\
(35.7)\end{array}$ & 0.12 & $\begin{array}{l}0.96(0.649 \\
, 1.428)\end{array}$ & 0.85 \\
\hline $\begin{array}{l}\text { Respiratory Failure Required } \\
\text { MV, n (\%) } \$ \star \$\end{array}$ & $\begin{array}{l}56 / 170 \\
(32.9)\end{array}$ & $\begin{array}{l}34 / 81 \\
(41.9)\end{array}$ & 0.16 & $\begin{array}{l}2.27(1.051 \\
, 4.898)\end{array}$ & 0.03 \\
\hline AKI during ICU stay, n (\%) & $\begin{array}{l}216 / 467 \\
(46.2)\end{array}$ & $\begin{array}{l}120 / 262 \\
(45.8)\end{array}$ & 0.91 & $\begin{array}{l}0.94(0.631 \\
, 1.405)\end{array}$ & 0.77 \\
\hline $\begin{array}{l}\text { Liver Injury during ICU stay, } n \\
\text { (\%) }\end{array}$ & $\begin{array}{l}49 / 465 \\
(10.5)\end{array}$ & $\begin{array}{l}28 / 262 \\
(10.6)\end{array}$ & 0.95 & $\begin{array}{l}0.97(0.539 \\
, 1.729)\end{array}$ & 0.91 \\
\hline \multirow[t]{2}{*}{$\begin{array}{l}\text { Thrombosis during ICU stay, } \\
\text { n (\%) }\end{array}$} & $\begin{array}{l}63 / 476 \\
(13.2)\end{array}$ & $\begin{array}{l}28 / 262 \\
(10.6)\end{array}$ & 0.31 & $\begin{array}{l}0.87(0.505 \\
, 1.490)\end{array}$ & 0.61 \\
\hline & & & & $\begin{array}{l}\text { beta } \\
\text { coefficient } \\
\text { (Estimates) } \\
(95 \% \mathrm{Cl})\end{array}$ & P-value \$* \\
\hline $\begin{array}{l}\text { MV duration during ICU stay } \\
\text { Days, Median }(\mathrm{Q} 1, \mathrm{Q} 3) \text { \&\# }\end{array}$ & $\begin{array}{l}9.0(4.0 \\
17.0)\end{array}$ & $\begin{array}{l}10.0(4.0 \\
18.0)\end{array}$ & 0.46 & $\begin{array}{l}0.06(-0.11 \\
0.22)\end{array}$ & 0.49 \\
\hline $\begin{array}{l}\text { ICU Length of Stay Days, } \\
\text { Median }(\mathrm{Q} 1, \mathrm{Q} 3)^{\text {\& }}\end{array}$ & $\begin{array}{l}8.0(5.0 \\
13.0)\end{array}$ & $\begin{array}{l}9.0(6.0 \\
15.0)\end{array}$ & 0.01 & $\begin{array}{l}0.12(-0.05 \\
, 0.29)\end{array}$ & 0.16 \\
\hline $\begin{array}{l}\text { Hospital Length of Stay } \\
\text { Days, Median }(\mathrm{Q} 1, \mathrm{Q} 3)^{\text {\& }}\end{array}$ & $\begin{array}{l}16.0 \\
(11.0 \\
26.0)\end{array}$ & $\begin{array}{l}18.0(13.0 \\
27.5)\end{array}$ & 0.04 & $\begin{array}{l}0.15(-0.00 \\
, 0.29)\end{array}$ & 0.05 \\
\hline
\end{tabular}




\section{Outcomes}

\section{Crude analysis}

Odds Ratio

(OR) $(95 \% \mathrm{Cl})$

P-value for

adjusted

$\begin{array}{lll}\text { Control } & \begin{array}{l}\text { Tocilizumab } \\ \text { group }\end{array} & \begin{array}{l}\mathrm{P} \text { - } \\ \text { value }\end{array}\end{array}$

$\Delta$ Denominator of the percentage is the total number of patients.

$\$ \star \$$ Denominator of the percentage is non-mechanically ventilated patients with 24 hours of ICU admission.

\&\# Denominator is patients who have respiratory failure requiring MV during ICU stay.

\& Denominator is patients who survived.

* T -Test / ${ }^{\wedge}$ Wilcoxon rank sum test is used to calculate the P-value.

${ }^{\wedge}$ Chi-square test is used to calculate the P-value.

** Fisher Exact test is used to calculate the P-value.

$\$ * *$ Multivariate Logistic regression is used after adjusting for patient's age, SOFA score, $\mathrm{PaO}_{2} / \mathrm{FiO}_{2}$ ratio baseline and systemic corticosteroids during ICU stay to calculate Odds ratio and p-value.

$\$^{*}$ Multivariate Logistic regression is used after adjusting for patient's age, SOFA score, $\mathrm{PaO}_{2} / \mathrm{FiO}_{2}$ ratio baseline and systemic corticosteroids during ICU stay to calculate estimates and p-value.

\section{Primary outcomes}

There was no significant difference in the incidence of microbial isolation ( $44.9 \%$ vs. $50.5 \%, p=0.14)$, emergence of resistant organisms $(58.1 \%$ vs. $64.2 \%, p=0.36)$ nor CRE detection $(4.4 \%$ vs. $3.8 \%, p=0.69)$ (Table 1). Moreover, after adjusting for possible confounders such as patients' comorbidities (i.e., DM, CKD on dialysis), history of hospitalization or invasive procedure within one year, history of antibiotics exposure in the last three months, systemic corticosteroids use during ICU, and ICU LOS, patients who received tocilizumab were not associated with higher incidence of microbial isolation (OR $1.34 ; 95 \% \mathrm{Cl}$, 0.91-1.94 p = 0.13), resistant organisms (OR 1.00; 95\% Cl, 0.51-1.98 p = 0.99) nor CRE detection (OR $0.67 ; 95 \% \mathrm{Cl}, 0.29-1.54 \mathrm{p}=0.34)$ (Table 1).

\section{Secondary outcomes}

In a crude analysis, there was no significant difference in the secondary outcome of in-hospital mortality $(44.7 \%$ vs. $46.9 \%, p=0.57)$ or 30 -day mortality $(35.7 \%$ vs. $41.6 \%, p=0.12)$ between the tocilizumab and the control group, respectively (Table 2). Additionally, in a multivariable logistic regression adjusting for age, baseline SOFA scores, $\mathrm{PaO2} / \mathrm{FiO} 2$ ratio, systemic corticosteroid use there was no difference in the inhospital mortality (OR $1.18 ; 95 \% \mathrm{Cl}, 0.79-1.76 \mathrm{p}=0.42)$ or 30 -day ICU mortality (OR $0.96 ; 95 \% \mathrm{Cl}, 0.65-$ $1.43 p=0.85$ ). Prior to adjustment, hospital length of stay ( 9 days vs. 8 days, $p=0.01$ ) and ICU length of stay ( 9 days vs. 8 days, $p=0.04$ ) were significantly higher in the tocilizumab group compared to the 
control group, respectively. However, they were not significant after adjusting for possible confounders (age, baseline SOFA score, $\mathrm{PaO}_{2} / \mathrm{FiO}_{2}$ ratio, and systemic corticosteroid use) (Table 2).

Among non-mechanically ventilated patients within 24 hours of ICU admission, there was a significant difference in the incidence of respiratory failure requiring MV between the two groups (OR $2.27 ; 95 \% \mathrm{Cl}$, $1.05-4.89 p=0.03$ ). On the flip side, there were no significant differences between the control and tocilizumab groups in terms of incidence of acute kidney injury, thrombosis, and duration of mechanical ventilation during ICU stay after adjusting for possible confounders (Table 2). The follow-up inflammatory markers were statistically significant among the groups, D-dimer and iron were higher in tocilizumab group but fibrinogen was lower in tocilizumab group. CRP and procalcitonin were similar among the groups, as described in Table 3.

Table 3

Follow-up for inflammatory markers

\begin{tabular}{|c|c|c|c|c|c|}
\hline \multirow[t]{2}{*}{ Outcomes } & \multicolumn{3}{|c|}{ Crude analysis } & \multirow{2}{*}{$\begin{array}{l}\text { beta coefficient } \\
\text { (Estimates) }(95 \% \mathrm{Cl})\end{array}$} & \multirow{2}{*}{$\begin{array}{l}\mathrm{P}- \\
\text { value } \\
\$^{*}\end{array}$} \\
\hline & $\begin{array}{l}\text { Control } \\
\text { group }\end{array}$ & $\begin{array}{l}\text { Tocilizumab } \\
\text { group }\end{array}$ & $\begin{array}{l}\text { P- } \\
\text { value }\end{array}$ & & \\
\hline $\begin{array}{l}\text { Serum iron level follow-up, } \\
\text { Median (Q1, Q3) }\end{array}$ & $\begin{array}{l}5.88 \\
(3.70 \\
10.23)\end{array}$ & $\begin{array}{l}8.91(3.98 \\
13.66)\end{array}$ & 0.004 & $0.31(0.15,0.47)$ & 0.0002 \\
\hline $\begin{array}{l}\text { D-dimer level follow-up } \\
\text { (mg/l), Median (Q1, Q3) }\end{array}$ & $\begin{array}{l}3.24 \\
(1.13 \\
8.51)\end{array}$ & $\begin{array}{l}4.01 \\
(1.53,14.74)\end{array}$ & 0.006 & $2.81(2.39,3.24)$ & $<.0001$ \\
\hline $\begin{array}{l}\text { Fibrinogen level follow-up } \\
(\mathrm{gm} / \mathrm{l}) \text {, Median }(\mathrm{Q} 1, \mathrm{Q} 3)\end{array}$ & $\begin{array}{l}6.29 \\
(4.59 \\
7.85)\end{array}$ & $\begin{array}{l}5.43(3.60, \\
7.30)\end{array}$ & 0.0003 & $-0.18(-0.28,-0.08)$ & 0.0002 \\
\hline $\begin{array}{l}\text { CRP level follow-up (mg/l), } \\
\text { Median (Q1, Q3) }\end{array}$ & $\begin{array}{l}164.0 \\
(84.0 \\
261.0)\end{array}$ & $\begin{array}{l}187.0(93.0 \\
302.0)\end{array}$ & 0.14 & $0.11(-0.05,0.27)$ & 0.18 \\
\hline $\begin{array}{l}\text { Procalcitonin level follow- } \\
\text { up (ng/ml), ), Median (Q1, } \\
\text { Q3) }\end{array}$ & $\begin{array}{l}0.46 \\
(0.13 \\
1.73)\end{array}$ & $\begin{array}{l}0.55(0.15, \\
3.32)\end{array}$ & 0.09 & $-0.37(-1.77,1.03)$ & 0.60 \\
\hline \multicolumn{6}{|c|}{ * T -Test / ^ Wilcoxon rank sum test is used to calculate the P-value. } \\
\hline \multicolumn{6}{|c|}{${ }^{\wedge \wedge}$ Chi-square test is used to calculate the P-value. } \\
\hline \multicolumn{6}{|c|}{ ** Fisher Exact test is used to calculate the P-value. } \\
\hline \multicolumn{6}{|c|}{$\begin{array}{l}\$^{*} \text { Multivariate Logistic regression is used after adjusting for patient's age, SOFA score, } \mathrm{PaO}_{2} / \mathrm{FiO}_{2} \\
\text { ratio baseline and systemic corticosteroids during ICU stay to calculate estimates and p-value. }\end{array}$} \\
\hline
\end{tabular}

\section{Discussion}


In our retrospective cohort study of critically ill COVID-19 patients, we found that patients who received tocilizumab did not have a significantly higher risk of acquiring multidrug-resistant bacteria such as MDR, XDR, PDR nor had higher risk of CRE. Moreover, the tocilizumab group had similar in-hospital mortality, 30 day ICU mortality, days on MV during ICU stay, ICU length of stay, and hospital length of stay to those who did not receive tocilizumab. However, patients who received tocilizumab did have a significantly higher risk of respiratory failure that required MV.

Our primary outcomes findings were observed after adjusting for the patient's comorbidities, history of hospitalization or invasive procedure within one year, history of antibiotics exposure in the last three months, systemic corticosteroids during ICU, and ICU LOS. The finding of no difference in secondary infections rate is similar to previous studies that conducted adjusted matched analyses for tocilizumab and controls and showed no difference between the arms only (26 in the treatment arm, 25 in the control arm; $95 \% \mathrm{Cl}$ for difference, -0.15 to $0.13 ; P=1.00)(20,21)$. This was also demonstrated in another multicenter cohort study of 4485 adults with COVID-19 admitted to (ICUs) has found no differences in secondary infection rate (140 [32.3\%] vs 1085 [31.1\%]) $(22,23)$. This is also concurrent with a recent meta-analysis that evaluated tocilizumab use in critically ill patients and found no difference in secondary infection between tocilizumab and the control group (24). However, other published trials found lower rates of secondary infections with tocilizumab $(7,10,11)$, while another study found higher rates of secondary infections with tocilizumab (25). The differences in outcomes in these studies could be attributed to the unadjusted analysis in some studies and if studied on critically ill patients or other hospitalized patients as critical care settings are more prone to infections. Further randomized studies are needed to confirm these findings.

We found no significant difference in 30-day ICU and in-hospital mortality rates between the two groups. Previous studies depicted mixed results in the mortality outcome. In one randomized control study comparing tocilizumab with usual care in non ICU COVID-19 patients with moderate to severe pneumonia, there was also no difference in mortality at 28-days (10). Similarly, a recent meta-analysis in ICU patients found no significant difference in mortality rates between the tocilizumab group and the comparator groups (24). Alternatively, a previous retrospective cohort study in ICU COVID-19 patients found significantly lower ICU mortality in the tocilizumab group but no difference in the 28-day mortality (23). Another retrospective cohort study for non-ICU patients found lower mortality with tocilizumab (25). In the recent RECOVERY trial that included non-ICU COVID-19 patients, there was a significant lower 28-days mortality in the tocilizumab group compared with placebo. However, it is important to note that this benefit may be attributed to the combination with corticosteroids, as more than $80 \%$ on included patients were recieveing corticosteroids at the same time, a confounder that we took in consideration and adjusted for it (26).

Furthermore, a randomized clinical trial evaluated tocilizumab versus usual care in ICU patients with COVID-19 and found no significant difference in 15-day, in-hospital and 28-days mortality between the two groups. However, this trial was prematurely interrupted after the first interim analysis due to an excess number of deaths at day 15 in the tocilizumab group (27). Overall, the mortality benefit with 
tocilizumab in an ICU setting is still debatable, but other potential clinical benefits could justify its use and improve COVID-19 patients course of illness.

Intersteningly, our study demonstrated that the progression of care towards the use of mechanical ventilator was higher among patients who received tocilizumab. This could be due to the increase in IL-6 levels and the cytokine storm shortly after the infusion, which was associated with worsening $\mathrm{PaO}_{2} / \mathrm{FIO}_{2}$ ratio and decline in the lung function as reported by Rossotti $\mathrm{R}$, et al (28). These finding are contradicted by the result of two randomized controlled trials conducted by Hermine O, et al (10) and Salama C, et al (6), which showed that the use of tocilizumab was associated with reduced risk of intubation and need for mechanical ventilation.

In this study, tocilizumab use was not associated with any statistically significant differences in the ICU and hospital length of stay. In contrast to our findings is the reports from COVACTA trial (5) were they found that the median time until patients were discharged from the hospital or was 20 days in the tocilizumab group and 28 days in the placebo group. Also, they report shorter ICU Los, the median duration of ICU stay was 9.8 days in the tocilizumab group and 15.5 days in the placebo group, for a difference of 5.8 days.

Among patient who received tocilizumab, we found differences in inflammatory markers levels compared with the control group. The D-dimer levels were higher in the tocilizumab group compared with placebo. In line with this results, Rossotti et al. found that $d$-dimer rise by day 5 , then it decreased but without returning to baseline values (28). This finding is consistent with previous observational studies $(30,31)$ were C-reactive protein, IL-6 improved; however, D-dimer levels increased significantly and is concerning as it might increase the risk of thromboembolic events, which is increased among patient with covid-19 (32). Fibrinogen levels during ICU stay were lower in the tocilizumab group, which was expected as tocilizumab tends to decrease inflammatory markers.

Our study has some limitations that need to be addressed. The retrospective and observational nature of the study puts the study at risk for residual confounding, despite adjustment for possible confounders. Furthermore, the decision to prescribe tocilizumab to COVID-19 patients was guided by the institutional and the Ministry of Health treatment protocols which continued to change with the emergence of new data. Moreover, the subsequent doses of tocilizumab and the timing between them could affect some of the clinical outcomes, which warrant further studies.

\section{Conclusion}

Overall, our study demonstrated that tocilizumab use in critically ill COVID-19 patients was not associated with different incidence of microbial isolation, emergence of resistant organisms, detection of CRE organisms, or mortality benefits. Tocilizumab was associated with an increased risk of respiratory failure requiring mechanical ventilation. Further randomized clinical and interventional studies are required to confirm our findings. 


\section{Abbreviations}

Intensive care units (ICUs), Coronavirus disease 2019 (COVID-19), Mechanical ventilation (MV), Length of Stay (LOS)

\section{Declarations}

\section{Acknowledgments}

Not applicable.

\section{Author contributions}

All authors contributed to data collections, analysis, drafted, revised, and approved the manuscript's final version.

\section{Funding}

None.

\section{Availability of data and material}

The datasets used and/or analyzed during the current study are available from the corresponding author on reasonable request.

\section{Ethics approval and consent to participate}

The study was approved by Unit of Biomedical Ethics Research Committee at faculty of medicine in King Abdulaziz University with Reference No 15-21. The second site was King Abdulaziz Medical City, Riyadh and was approved by King Abdullah International Medical Research Center in February 2021

(Ref.\#NRC21/025/01). All methods were performed under relevant guidelines and regulations. Waiver for consents was waived from both center based on the retrospective chart review nature of the study.

Participants' confidentiality was strictly observed throughout the study by using the anonymous unique serial number for each subject and restricting data only to the investigators. Informed consent was waived because of the study's retrospective nature, and the analysis used anonymous clinical data as per the policy of the local research center.

\section{Consent for publication}


Not applicable.

\section{Competing interests}

No author has a conflict of interest in this study.

\section{References}

1. COVID-19 Dashboard by the Center for Systems Science and Engineering (CSSE) at Johns Hopkins University. Retrieved 1 February 2021.

2. Wiersinga WJ, Rhoades A, Cheng A, Peacock S, Prescott H. Pathophysiology, transmission, diagnosis, and treatment of coronavirus disease 2019 (COVID-19): a review. JAMA 2020;324:782e93.

3. Clinical features of patients infected with 2019 novel coronavirus in Wuhan, China. PubmedID 31986264

4. hen X, Zhao B, Qu Y, Chen Y, Xiong J, Feng Y, et al. Detectable serum SARSCoV-2 viral load (RNAaemia) is closely correlated with drastically elevated interleukin 6 (IL-6) level in critically ill COVID-19 patients. Clin Infect Dis 2020;71:1937e42. https://doi.org/10.1093/cid/ciaa449.

5. Rosasl, BräuN, WatersM, et al. Tocilizumab in hospitalized patients with COVID-19 pneumonia. February 25, 2021. DOI: 10.1056/NEJMoa2028700

6. SalamaC, HanJ, YauL, et al. Tocilizumab in patients hospitalized with Covid-19 pneumonia. N Engl J Med. 2021;384:20-30.

7. SalvaraniC, DolciG, MassariM, et al. Effect of tocilizumab vs. standard care on clinical worsening in patients hospitalized with COVID-19 pneumonia: a randomized clinical trial. JAMA Intern Med. 2021;181:24-31

8. McCreary EK, Pogue JM. Coronavirus disease 2019 treatment: a review of early and emerging options. Open Forum Infect Dis. 2020;7(4):ofaa105.

9. Sebba A. Tocilizumab: the first interleukin-6-receptor inhibitor. Am J Health Syst Pharm. 2008 Aug 1;65(15):1413-8. doi: 10.2146/ajhp070449. PMID: 18653811.

10. HermineO, MarietteX, TharauXPL, et al. Effect of tocilizumab vs. usual care in adults hospitalized with COVID-19 and moderate or severe pneumonia: a randomized clinical trial. JAMA Intern Med. 2021;181:32-40.

11. StoneJH, FrigaultMJ, Serling-BoydNJ, et al. Efficacy of tocilizumab in patients hospitalized with Covid-19. N Engl J Med. 2020;383:2333-2344.

12. Tocilizumab in patients with severe COVID-19: a retrospective cohort study. PMID: 32835257

13. Porretta AD, Baggiani A, Arzilli G, Casigliani V, Mariotti T, Mariottini F, Scardina G, Sironi D, Totaro M, Barnini S, Privitera GP. Increased Risk of Acquisition of New Delhi Metallo-Beta-Lactamase-Producing Carbapenem-Resistant Enterobacterales (NDM-CRE) among a Cohort of COVID-19 Patients in a 
Teaching Hospital in Tuscany, Italy. Pathogens. 2020 Aug 5;9(8):635. doi: 10.3390/pathogens 9080635 .

14. Aleidan FAS, Alkhelaifı $\mathrm{H}$, Alsenaid $\mathrm{A}$, et al. Incidence and risk factors of carbapenem-resistant Enterobacteriaceae infection in intensive care units: a matched case-control study [published online ahead of print, 2020 Sep 20]. Expert Rev Anti Infect Ther. 2020;1-6.

15. The Clinical and Laboratory Standards Institute (CLSI) M60 ED2:2020 edition. (Accessed 02/09/2021)

16. Lin CY, Chen YC. Acute kidney injury classification: AKIN and RIFLE criteria in critical patients. World J Crit Care Med. 2012;1(2):40-45. Published 2012 Apr 4. doi:10.5492/wjccm.v1.i2.40

17. ICD - ICD-10-CM - International Classification of Diseases, Tenth Revision, Clinical Modification. Cdc.gov. https://www.cdc.gov/nchs/icd/icd10cm.htm. Published 2021. Accessed January 29, 2021.

18. Khalid Al Sulaiman, Alaa Alhubaishi, Ohoud Al Juhani et al. Early Versus Late Use of Dexamethasone in Critically III Patients With COVID-19: A Multicenter, Prospective Cohort Study. May 1, 2021, PREPRINT (Version 1) available at Research Square [https://www.researchsquare.com/article/rs349677/v1]

19. Magiorakos AP, Srinivasan A, Carey RB, et al. Multidrug-resistant, extensively drug-resistant and pandrug-resistant bacteria: an international expert proposal for interim standard definitions for acquired resistance. Clin Microbiol Infect. 2012;18(3):268-281. doi:10.1111/j.14690691.2011.03570.x

20. Ignatius EH, Wang K, Karaba A, Robinson M, Avery RK, Blair P, Chida N, Jain T, Petty BG, Siddiqui Z, Melia MT, Auwaerter PG, Xu Y, Garibaldi BT. Tocilizumab for the Treatment of COVID-19 Among Hospitalized Patients: A Matched Retrospective Cohort Analysis. Open Forum Infect Dis. 2020 Dec 28;8(1):ofaa598. doi: 10.1093/ofid/ofaa598.

21. Rajendram P, Sacha GL, Mehkri O, Wang X, Han X, Vachharajani V, Duggal A. Tocilizumab in Coronavirus Disease 2019-Related Critical IIIness: A Propensity Matched Analysis. Crit Care Explor. 2021 Jan 13;3(1):e0327. doi: 10.1097/CCE.0000000000000327.

22. Campochiaro C, Della-Torre E, Cavalli G, et al. Efficacy and safety of tocilizumab in severe COVID-19 patients: a single-centre retrospective cohort study. Eur J Intern Med 2020; 76:43-9

23. Gupta S, Wang W, Hayek SS, et al. Association between early treatment with tocilizumab and mortality among critically ill patients with COVID-19. JAMA Intern Med 2020; e206252.

24. Soheila Rezaei, Behzad Fatemi, Zahra Karimi Majd, Hossein Minaei, Mohammad Peikanpour, Nassim Anjidani, Ali Taheri, Farzaneh Dastan \& Reza Mosaed(2021) Efficacy and safety of Tocilizumab in severe and critical COVID-19: A Systematic Review and Meta-Analysis, Expert Review of Clinical Immunology, DOI: 1080/1744666X.2021.1908128

25. Guaraldi G, Meschiari M, Cozzi-Lepri A, et al. Tocilizumab in patients with severe COVID-19: a retrospective cohort study. Lancet Rheumatol 2020; 2:e474-84.

26. Horby PW, Pessoa-Amorim G, Peto L, Brightling CE, Sarkar R, Thomas K et al. Tocilizumab in patients admitted to hospital with COVID-19 (RECOVERY): preliminary results of a randomised, controlled, 
open-label, platform trial. medRxiv. 2021:2021.02.11.21249258. doi:10.1101/2021.02.11.21249258.

27. Veiga VC, Prats JAGG, Farias DLC, et al. Effect of tocilizumab on clinical outcomes at 15 days in patients with severe or critical coronavirus disease 2019: randomised controlled trial. BMJ. 2021;372:n84. doi:10.1136/bmj.n84

28. Rossotti R, Travi G, Ughi N, et al. Safety and efficacy of anti-il6-receptor tocilizumab use in severe and critical patients affected by coronavirus disease 2019: a comparative analysis. J Infect. 2020;81(4):e11-e17

29. https://www.cdc.gov/coronavirus/2019-ncov/long-term-effects.html.Accessed on 25April,2021.

30. Price, Christina C et al. "Tocilizumab Treatment for Cytokine Release Syndrome in Hospitalized Patients With Coronavirus Disease 2019: Survival and Clinical Outcomes." Chest vol. 158,4 (2020): 1397-1408. doi:10.1016/j.chest.2020.06.006

31. Atallah, B., El Nekidy, W., Mallah, S.I. et al.Thrombotic events following tocilizumab therapy in critically ill COVID-19 patients: a Façade for prognostic markers. Thrombosis J 18, 22 (2020). https://doi.org/10.1186/s12959-020-00236-9

32. Bikdeli B, Madhavan MV, Jimenez D, Chuich T, et al. Global COVID-19 Thrombosis Collaborative Group, Endorsed by the ISTH, NATF, ESVM, and the IUA, Supported by the ESC Working Group on Pulmonary Circulation and Right Ventricular Function. COVID-19 and Thrombotic or Thromboembolic Disease: Implications for Prevention, Antithrombotic Therapy, and Follow-Up: JACC State-of-the-Art Review. J Am Coll Cardiol. 2020 Jun 16;75(23):2950-2973. doi: 10.1016/j.jacc.2020.04.031.

\section{Supplementary Files}

This is a list of supplementary files associated with this preprint. Click to download.

- Additionalfile1DemographyandBaselinecharacteristics.docx

- Additionalfile2Coexistingillness.docx 